\title{
Platelet-rich plasma as a treatment for chronic patellar tendinopathy: comparison of a single versus two consecutive injections
}

\author{
Rachad Zayni ${ }^{1}$ \\ Mathieu Thaunat ${ }^{2}$ \\ Jean-Marie Fayard ${ }^{2}$ \\ Jean-Philippe Hager ${ }^{2}$ \\ Yannick Carrillon ${ }^{2}$ \\ Julien Clechet $^{2}$ \\ François Gadea ${ }^{2}$ \\ Pooler Archbold ${ }^{2}$ \\ Bertrand Sonnery Cottet $^{2}$
}

1 Department of Orthopedic Surgery, Saint-Vincent Hospital, Université Catholique de Lille, France

2 Générale de Santé, Hôpital Privé Jean Mermoz, Centre Orthopédique Santy, Lyon, France

Corresponding author:

Rachad Zayni

Department of Orthopedic Surgery, Saint-Vincent Hospital

B.P. 387 Boulevard de Belfort

59020 Lille, France

E-mail: drzayni@gmail.com

\section{Summary}

Background: platelet-rich-plasma is increasingly used in chronic patellar tendinopathy. Ideal number of PRP injections needed is not yet established. This study compares the clinical outcomes of a single versus two consecutive PRP injections.

Method: between December 2009 and January 2012, 40 athletes with proximal patellar tendinopathy were treated by PRP injection. Patients received single (20 patients) or two PRP injections 2 weeks apart (20 patients). All patients underwent prospective clinical evaluation, including Victorian Institute of Sport Assessment-Patella (VISA-P) score, visual analog scale (VAS) for pain, and Tegner scale before PRP and after a minimum of 2 year follow-up.

Results: 9 patients failed PRP treatment and needed surgery. 1 patient was lost to follow-up. For the remaining patients, the VISA-P, VAS, and Tegner scores all significantly improved from $\mathbf{3 5 . 2}$ to $\mathbf{7 8 . 5}$ $(p=0.0001), 6.6$ to $2.4(p=0.0001)$, and 4.8 to 6.9 ( $p$ $=0.0003$ ). Patients who received two injections had better scores than those who received single injection with VAS of 1.07 versus 3.7 ( $p=0.0005)$, Tegn- er score of 8.1 versus $5.9(p=0.0003)$ and VISA-P of 93.2 versus $65.7(p=0.0001)$.

Conclusions: two consecutive PRP injections in chronic patellar tendinopathy showed better improvement in outcomes when compared to single injection.

Level of evidence: randomized prospective consecutive series, Level 2.

KEY WORDS: conservative treatment, eccentric exercises, patellar tendon, platelet-rich-plasma, tendinopathy.

\section{Introduction}

Chronic patellar tendinopathy (PT) remains a challenging problem for both orthopedic surgeons and sport medicine practitioners. It is often caused by overuse of the knee extensors in activities such jumping, running and kicking. Clinically, this tendinopathy is characterized by anterior knee pain and focal tenderness at the distal border of the patella. It affects athletes in numerous sports, at all levels of participation and can severely limit performance ${ }^{1}$. The precise etiology of this tendinopathy is not clear. Several histopathological studies have shown a degenerative process with tendon thickening, disturbed collagen distribution, neovascularization and increased cellularity with incompletely healed tendon micro-ruptures ${ }^{2-5}$.

There is currently no consensus on its optimal treatment. Conservative management is the first line treatment including eccentric physical therapy, icing, activity modification, and non-steroidal anti-inflammatory drugs (NSAIDs)6,7. Despite conservative treatment, patellar tendinopathy may remain resistant to therapy and can often lead to a prolonged clinical course.

Autologous PRP intra-lesional injection is being used increasingly in the treatment of many soft tissue injuries. Recently, there has been great interest in using PRP for sports related injuries, particularly patellar tendinopathy ${ }^{8}$, although the rationale for use PRP is still unclear ${ }^{9}$. It is hypothesized that the growth factors and other bioactive molecules released by the alpha granules in platelets present in PRP have the potential to enhance cellular migration, proliferation, angiogenesis, and matrix deposition in tendon healing ${ }^{10-13}$.

To date, the ideal protocol of PRP application concerning the volume, number and timing of injections has not been established ${ }^{14}$. An animal model study 
Platelet-rich plasma as a treatment for chronic patellar tendinopathy: comparison of a single versus two consecutive injections

performed by Parafioriti et al. had demonstrated that a single injection of PRP in Achilles tendinopathy is limited in efficacy and dissipate after one week ${ }^{9}$.

The goal of our study was to compare the clinical outcomes of a single versus two consecutive ultrasound (US)-guided PRP injections in patients with chronic patellar tendinopathy who had failed 3 months of conservative management. Our hypothesis was that patients who receive two PRP injections have a better outcome than those who receive a single injection.

\section{Materials and methods}

\section{Selection of patients}

In December 2009, a prospective study was conducted in our institution to assess patients who underwent PRP injection for patellar tendinopathy. Inclusion criterion was chronic anterior knee pain with focal tenderness at the proximal insertion of patellar tendon on clinical examination. Diagnosis was performed by an attending orthopedic surgeon and was confirmed by $M R I$ read by an attending radiologist. MRI showed enhanced signal intensity in T2 sequences near the distal pole of patella with thickening of the tendon at this level (Fig. 1A). In all patients, a same nonoperative treatment protocol had been followed for at least 3 months and had failed. This conservative treatment consisted of rest, non-steroidal anti-inflammatory drugs (NSAIDs), eccentric exercises (Stanish proto$\mathrm{col})^{15}$ and extracorporeal shock wave therapy. Exclusion criteria were coexisting knee lesions, knee surgery or corticosteroid injection in the previous 3 months. Patients were recruited from December 2009 to January 2012. During this time period, 242 patients were treated in our institution by autologous PRP injection for different tendinopathies. Fifty of them (20.6\%) suffered from proximal patellar tendinopathy and were assessed for eligibility. Ten of these patients were excluded (4 patients with previous ipsilateral ACL reconstruction, 3 with a previous corticosteroid injection and 3 with ipsilateral patellar chondropathy on MRI examination). The remaining 40 patients were eligible
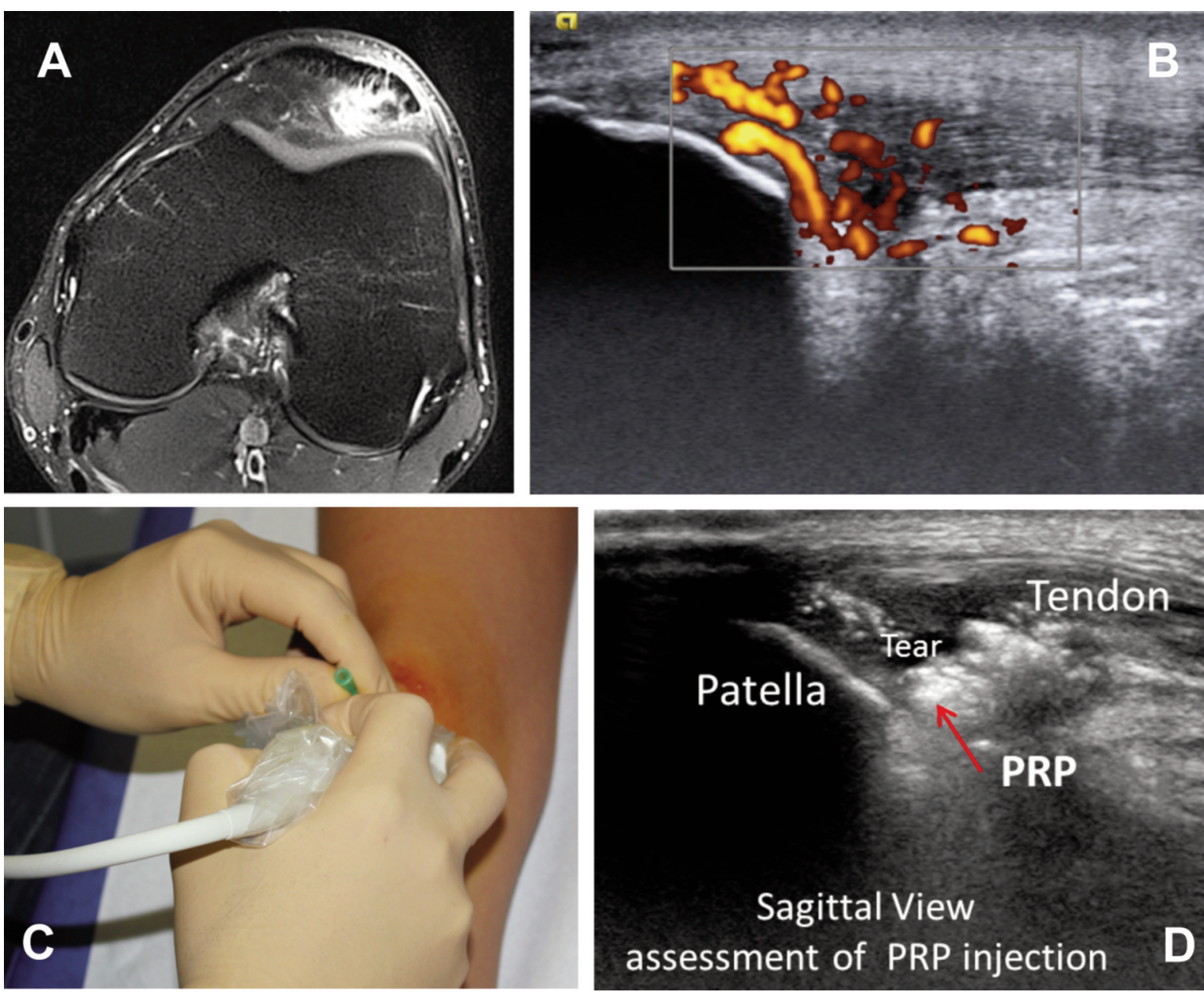

Figure 1. A) MRI (axial view) showing enhanced signal intensity in T2 sequences near the distal pole of patella with thickening of the tendon at this level. B) Ultrasound aspect of the tendon lesion. C) Ultrasound guided PRP injection procedure: knee was held in extension position to relax tendon fibers and to facilitate PRP diffusion. D) Ultrasound assessment of PRP injection. 


\section{R. Zayni et al.}

for the study. These patients were randomized, using a coin toss, to receive a single PRP injection (group A, $n=20$ patients) or two PRP injections 2 weeks apart (group $\mathrm{B}, \mathrm{n}=20$ patients) (Flowchart 1 ).

All patients were asked to avoid NSAIDs 4 weeks before and after the treatment. Informed consent was obtained for all participants. The study protocol was approved by the local ethical committee and meets the standards and lows of MLT journal as described by Padulo et al. ${ }^{16}$.

\section{Ultrasound-guided PRP injection}

PRP was prepared using the Arthrex ACP system (Arthrex Inc, Naples, Florida, USA). In this preparation, platelet concentration is known to be two times higher that of whole blood with no leukocytes. Therefore, growth factors are significantly more concentrated in the ACP than in whole blood17,18. A $15 \mathrm{ml}$ of venous blood was drawn by a registered nurse into the Arthrex double syringe system. After centrifugation at $1700 \mathrm{rpm}$ for 5 minutes, a final volume of $6 \mathrm{ml}$ of pure
PRP was obtained and no activating agent was added. The same trained radiologist, using a 22gauge needle, then immediately injected the PRP under sterile conditions. Injections were performed under ultrasonography control within and around the hypoechogenic tendon area (Figs. 1B, 1D). The knee was held in extension during the injection procedure to relax the tendon fibers to facilitate PRP diffusion into the injured area (Fig. 1C).

\section{Rehabilitation protocol}

Rest was prescribed for two weeks after the last PRP injection. Then all patients, supervised by a physical therapist, followed a same standardized rehabilitation program, 3 times a week for 2 months. Treatment plans focused on stretching, electrostimulation, cycling and eccentric strengthening, as described by Stanish et al. ${ }^{15}$ and Kaux et al. ${ }^{19}$. After then, patients had to continue auto-reeducation for more 2 months which followed by maintenance exercises. Patients were authorized to resume sport as tolerated.

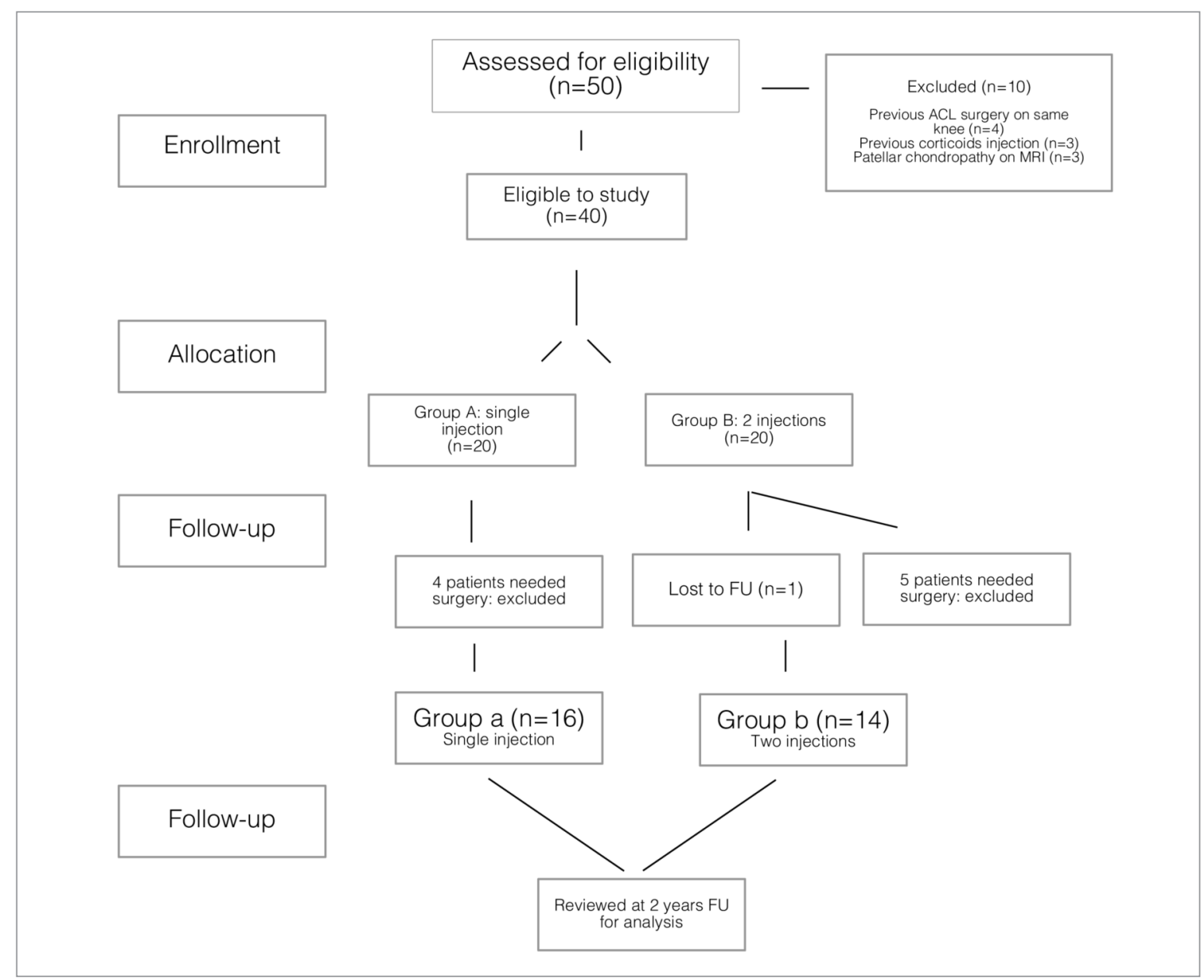

Flowchart 1. Enrollment of patients in the study.

FU: follow-up; $\mathrm{ACL}$ : anterior cruciate ligament 
Platelet-rich plasma as a treatment for chronic patellar tendinopathy: comparison of a single versus two consecutive injections

\section{Clinical outcomes evaluation}

The Victorian Institute of Sport Assessment-Patella (VISA-P) score, visual analog scale (VAS) for pain, and Tegner activity scale were used for clinical evaluation. The VISA score is a validated questionnaire that can be used to quantitatively assess severity of symptoms in patients with $\mathrm{PT}^{20}$. VAS pain scale is useful to adopt as a complement to the VISA-P and is well validated in patellar tendinopathy ${ }^{18}$. Clinical scores were noted before the procedure. Follow-up was begun 2 months after the last injection, and every 4 months with a 2-year minimum follow-up.

At the 2-month follow-up, patients of group $A$ that failed PRP treatment were not included to a second intention-to-treat analysis and were forwarded to surgical treatment without proposing a second PRP injection.

\section{Statistical Analysis}

Descriptive statistics were used to present data of the series at baseline. Comparison was made between the pre- and post-injection for all clinical scores with the Wilcoxon signed rank test and results at 2 years of reviewed patients of group A and B were compared using the student $t$ test. Level of statistical significance was set at $p<0.05$. All statistical analysis was carried out with SPSS 21.0.

\section{Results}

A total of 40 patients was enrolled in this study between December 2009 and January 2012. At the 2 month follow-up, 4 patients in group $A$ and 5 patients in group $B$ noticed no clinical improvement after PRP treatment and rehabilitation protocol $(22.5 \%$ of pa- tients). The mean VISA-P, VAS and Tegner scores of these 9 patients at baseline and at the 2-month follow-up were unchanged [respectively 35.7 versus $48.8(p=0.035), 6.1$ versus $5.4(p=0.094)$ and 5.6 versus $5.7(p=0.27)$ ]. Failure rate was similar in the two group A and B (respectively 20 and 26\%, $p=$ $0.6)$. When compared to the rest of the series, these 9 patients had similar baseline scores. They underwent surgical treatment and therefore were excluded from the final analysis. One patient from group $B$ was lost to follow-up (FU) and all the remaining 30 patients (group $a, n=16$ and group $b, n=14$ ) were reviewed after a minimum of 24-month follow-up. The mean age of these patients was 24.4 years (SD 3.3). All patients were athletes (17 elite athletes, $13 \mathrm{com}$ petitive non-elite athletes). Mean follow-up was 34 months (SD 7.8). All patients were graded 3B according to the classification of Blazina ${ }^{21}$ (unable to participate in sports at the same level as before the onset of symptoms).

Table 1 shows the characteristics of the population in the study. Mean delay between symptoms and PRP injection was 4.7 months (SD 1.2).

For all the 30 patients and at a mean of 34 month follow-up, the mean VISA-P score improved from 35.2 to $78.5(p<0.0001)$, mean VAS decreased from 6.6 to $2.4(p<0.0001)$, and mean Tegner knee score rose from 4.8 to $6.9(p<0.0003)$. 26 of the 30 patients $(86 \%)$ returned to their pre-symptom sporting level after a mean delay of 6.7 weeks (SD 2.4). This delay was similar in both groups $(p=0.13)$. The 4 remaining patients returned to a lesser activity level.

This sporting level recovery was maintained until the last follow-up. No post-injection complication was encountered in the series and no recurrence or progression of symptoms was noted during follow-up. Mean VISA, Tegner and VAS scores at baseline and last follow-up are summarized in Table 2.

Table 1. Characteristics of the patients in group a and group b.

\begin{tabular}{lll}
\hline & Group a $(\mathrm{n}=16)$ & Group $\mathrm{b}(\mathrm{n}=14)$ \\
\hline Age, $\mathrm{y}$, mean (SD) & $24.6(3.4)$ & $24.1(3.3)$ \\
\hline Sex ratio (M/F) & $14 / 2$ & $12 / 2$ \\
\hline Delay before injection, mo, mean (SD) & $4.9(1.3)$ & $4.4(1.06)$ \\
\hline Type of sport & & 1 \\
Handball & 2 & 1 \\
Basketball & 2 & 1 \\
Volleyball & 2 & 3 \\
Ski & 1 & 1 \\
Soccer & 3 & 2 \\
Jogging & 1 & 2 \\
Rugby & 1 & 1 \\
Athletics & 2 & 1 \\
Cycling & 1 & 1 \\
Tennis & 1 & \\
\hline Level of sport & & 6 \\
Elite athletes & 9 & \\
Competitive non-elite athletes & 7 & \\
\hline
\end{tabular}

SD: Standard Deviation 


\section{Comparison of group a and group b}

Demographic data, delay of symptoms to injection, and baseline clinical scores were all similar between the two groups a and $b$ (Tabs. 1, 3).

Student t-test was performed to compare clinical outcomes of the 2 groups a and b. Patients that received 2 PRP injections had significantly better clinical scores than those who received a single PRP injection with VAS of 1.07 versus $3.6(p=0.0005)$, Tegner score of 8.1 versus $5.9(p=0.0003)$ and VISA-P of 93.2 versus $65.7(p=0.0001)($ Tab. 3).

\section{Discussion}

The principal finding of this study was that patients who received two consecutive PRP injections 2 weeks apart had significantly better VAS, Tegner and VISA$P$ scores than those who received a single injection. A number of papers have previously reported the results of multiple injections ${ }^{14,22}$ but, to our knowledge, there have been no previous studies comparing a one PRP injection to a two PRP injection protocol. Most growth factors contained in platelets are shortlived and the benefit of PRP may dissipate over time ${ }^{9}$ 23. Therefore, we hypothesized that repeating PRP administration may have better outcomes in chronic patellar tendinopathy.

Patellar tendinopathy is a common disorder that can affect athletes in different sports at all levels of activity. Non-operative management is the mainstay of treatment including physical therapy, icing, activity modification, and NSAIDs ${ }^{6}$. Decline eccentric squat exercises are considered the primary line of treatment of $\mathrm{PT}^{6,7}$. Persistent cases may be additionally treated with ultrasound, shockwave therapy and sclerosing injections ${ }^{3,24,25}$. Recently, some Authors recommend Intratissue percutaneous electrolysis (EPIß) in combination with eccentric exercises in an attempt to stimulate healing response with promising results $^{26}$. Other Authors propose high volume injections in the retro-tendinous fat pad to provoke obliteration of the neovascularization in the pathological tendon?. The nonoperative treatment of patellar tendinopathy may not be effective in all cases despite several months of treatment. Surgical intervention is usually reserved for persistent symptoms after failure of nonoperative management ${ }^{27}$. When surgical treatment is indicated, arthroscopic methods have been found to be as affective as and safer then open techniques with satisfactory results and fast recovery rate ${ }^{28}$.

PRP intralesional injection is being used increasingly in the treatment of many soft tissue injuries. PRP is an autologous blood fraction rich in platelets and their associated growth factors. When injected at the site of muscle or tendon injury, it will act as an adjuvant to the natural healing process ${ }^{29}$. In addition, PRP is perceived to have a low side effect profile given its autologous origin. PRP can stimulate tendon healing through collagen regeneration and a well-ordered angiogenesis mediated by the release of many of these growth factors contained in the alpha granules in platelets ${ }^{30}$. The use of PRP in the treatment of tendon lesions over several years has led to a significant improvement in healing $11,12,30$. Thus, PRP treatment is currently proposed as an alternative treatment before surgery in persistent tendinopathy.

Question about how many PRP injections are needed remains unanswered. To date, no consensus on the minimal number of PRP injections that optimizes tendon healing has been published. Most growth factors contained in platelets are short-lived and thus, repeated administration is advised ${ }^{9}$.

Dragoo et al. ${ }^{23}$ in a randomized controlled study have proved that a single injection of PRP accelerates the recovery from patellar tendinopathy relative to exercise and ultrasound-guided dry needling alone, but

Table 2. VAS, Tegner and VISA-P scores at baseline and after 34 month mean follow-up for all the 30 patients.

\begin{tabular}{llll}
\hline Scores of the $\mathbf{3 0}$ patients & At baseline & At $\mathbf{3 4}$ month mean FU & $\boldsymbol{p}$ value (wilcoxon test) \\
\hline VAS (SD) & $6.6(1.4)$ & $2.4(2.12)$ & $<0.0001$ \\
\hline Tegner score (SD) & $4.8(1.3)$ & $6.9(1.8)$ & 0.0003 \\
\hline VISA-P score (SD) & $35.2(10)$ & $78.5(21.4)$ & $<0.0001$
\end{tabular}

Table 3. Comparison of clinical outcomes of group a and group b before PRP treatment and at the final follow-up; ns: non significant.

\begin{tabular}{lllllll}
\hline \multicolumn{3}{l}{ At baseline } & \multicolumn{3}{l}{ At 34 month mean FU } \\
\hline Clinical scores & VAS (SD) & Tegner score (SD) & VISA-P (SD) & VAS (SD) & Tegner score (SD) & VISA-P (SD) \\
\hline $\begin{array}{l}\text { Group a: } \\
\text { 1 PRP injection }\end{array}$ & $7.1(1.6)$ & $4.1(1.3)$ & $36.7(10.6)$ & $3.6(1.2)$ & $5.9(5.9)$ & $65.7(19.8)$ \\
\hline $\begin{array}{l}\text { Group b: } \\
\text { 2 PRP injections }\end{array}$ & $6.7(1.7)$ & $4.8(0.94)$ & $35.7(9.4)$ & $1.07(1.5)$ & $8.1(1.7)$ & $93.2(14)$ \\
\hline $\boldsymbol{p}$ value & $\mathrm{ns}$ & $\mathrm{ns}$ & $\mathrm{ns}$ & 0.0005 & 0.0003 & $<0.0001$ \\
\hline
\end{tabular}


Platelet-rich plasma as a treatment for chronic patellar tendinopathy: comparison of a single versus two consecutive injections

they remarked that the benefit of PRP dissipates over time. Other clinical studies suggest that a weekly repeated injection of PRP permitted better clinical outcomes $^{22,31}$. Charousset et al. ${ }^{14}$ studied a series of 28 athletes and demonstrated that the application of 3 consecutive US-guided PRP injections significantly improved the symptoms and function in athletes with chronic PT and allowed a more rapid recovery to their pre-symptom level of sporting participation. In addition, they found a return to a normal architecture of the patellar tendon after this treatment on MRI assessment. Filardo et al. ${ }^{22}$ treated 15 patients with multiple PRP injections and observed a statistically significant improvement in knee function and quality of life, and most patients had a good recovery and returned to their previous sporting activity level.

Our results corroborate with other reports of the literature supporting the efficacy of US-guided intratendinous PRP injection in improving clinical symptoms and function in patients suffering of chronic patellar tendinopathy.

Almost $23 \%$ of our patients had failed PRP treatment and needed surgery. This rate is similar to that of Charousset study ${ }^{14}$ that had found a failure in $21.5 \%$ of cases. Contrariwise, Dragoo et al. ${ }^{23}$ noted no failure in their PRP treatment group. This relatively high rate of failure might be due to the long delay before proposing PRP treatment. To date, PRP is not yet a routine practice. Its use depends on the habits and experience of physicians. Giving its autologous and low side effect profile, a more precocious management with PRP after the onset of PT may be a solution to enhance response and to lower the failure rate. Further studies are needed to elucidate this point.

The major limitation of this study was the lack of a control group in the assessment of the effectiveness of PRP. Although our study aimed mainly to compare the efficacy of one versus two PRP injections in PT, a control group with normal saline injection might be useful to reduce this limitation. Because we practice in private hospital, this control group was difficult to ensure. Another limitation was that neither MRI nor ultrasound follow-up was performed to document tendon healing after PRP injection and consequently this study lacks of correlation between imaging findings and outcomes. In addition, we did not measure the cellular content or the growth factors in the PRP preparation. Such assessment may be interesting to identify the optimal concentration of platelets for tendon healing and might be more useful than number of injections in understanding PRP effectiveness. Other limitations are the relatively short follow-up and low number of patients. A longer follow-up and larger series would be necessary for more meaningful conclusions. Finally, excluding patients who failed PRP treatment and needed surgery may introduce a bias in the assessment of clinical score improvement since we did not take their low scores into consideration. Failure rate was similar in the two groups $A$ and B. As these patients had a similar baseline score compared to the rest of the series, we excluded them from final analysis in attempt to have a single variable which would be easy to compare.

\section{Conclusions}

At a 34 month mean follow-up, patients suffering from chronic patellar tendinopathy who underwent two consecutive ultrasound-guided intratendinous PRP injections showed a better improvement in their outcomes when compared to a single injection. PRP injection improved clinical outcomes in almost $77 \%$ of patients and allowed them to return to their pre-symptom activity level in $86 \%$ of cases. Further studies with longer follow-up and larger series are necessary to establish the ideal protocol of PRP application concerning the volume, preparation, number and optimal timing of injections.

\section{Conflict of interest}

No conflict of interest.

\section{References}

1. Kettunen JA, Kvist M, Alanen E, Kujala UM. Long-term prognosis for jumper's knee in male athletes. A prospective followup study. Am J Sports Med. 2002;30:689-692.

2. Maffulli N, Khan KM, Puddu G. Overuse tendon conditions: time to change a confusing terminology. Arthroscopy. 1998; 14(8):840-843.

3. Alfredson $\mathrm{H}$, Ohberg L. Neovascularisation in chronic painful patellar tendinosis - promising results after sclerosing neovessels outside the tendon challenge the need for surgery. Knee Surg Sports Traumatol Arthrosc. 2005;13:74-80.

4. Gisslen K, Alfredson $\mathrm{H}$. Neovascularisation and pain in jumper's knee: a prospective clinical and sonographic study in elite junior volleyball players. Br J Sports Med. 2005;39:423428.

5. Knobloch K. The role of tendon microcirculation in Achilles and patellar tendinopathy. J Orthop Surg Res. 2008;30:18.

6. Larsson ME, Kall I, Nilsson-Helander K. Treatment of patellar tendinopathy - a systematic review of randomized controlled trials. Knee Surg Sports Traumatol Arthrosc. 2011;20:16321646.

7. Morton S, Chan O, King J, et al. High volume image-guided Injections for patellar tendinopathy: a combined retrospective and prospective case series. MLTJ. 2014;4(2):214-219.

8. Foster TE, Puskas BL, Mandelbaum BR, Gerhardt MB, Rodeo SA. Platelet-rich plasma: from basic science to clinical applications. Am J Sports Med. 2009;37:2259-2272.

9. Parafioriti A, Armiraglio E, Del Bianco S, Tibalt E, Oliva F, Berardi AC. Single injection of platelet-rich plasma in a rat Achilles tendon tear model. MLTJ. 2011;1(2):41-47.

10. McCarrel T, Fortier L. Temporal growth factor release from platelet-rich plasma, trehalose lyophilized platelets, and bone marrow aspirate and their effect on tendon and ligament gene expression. J Orthop Res. 2009;27:1033-1042.

11. Podesta L, Crow SA, Volkmer D, Bert T, Yocum LA. Treatment of partial ulnar collateral ligament tears in the elbow with platelet-rich plasma. Am J Sports Med. 2013;41:1689-1694.

12. Schnabel LV, Mohammed HO, Miller BJ. Platelet rich plasma (PRP) enhances anabolic gene expression patterns in flexor 
digitorum superficialis tendons. J Orthop Res. 2007;25:230240.

13. Trippel SB. Growth factors as therapeutic agents. Instr Course Lect. 1997;46:473.

14. Charousset C, Zaoui A, Bellaiche L, Bouyer B. Are multiple Platelet-Rich Plasma injections useful for treatment of chronic patellar tendinopathy in athletes? A prospective study. Am J Sports Med. 2014;42(4):906-911.

15. Stanish WD, Rubinovich RM, Curwin S. Eccentric exercise in chronic tendinitis. Clin Orthop Relat Res. 1986;208:65-68.

16. Padulo J, Oliva F, Frizziero A, Maffulli N. Muscles, Ligaments and Tendons Journal. Basic principles and recommendations in clinical and field science research. MLTJ. 2013;4:250-252.

17. Borzini $P$, Mazzucco L. Tissue regeneration and in loco administration of platelet derivatives: clinical outcome, heterogeneous products, and heterogeneity of the effector mechanisms. Transfusion. 2005;45:1759-1767.

18. Khan KM, Visentini PJ, Kiss ZS, et al. Correlation of ultrasound and magnetic resonance imaging with clinical outcome after patellar tenotomy: prospective and retrospective studies. Victorian Institute of Sport Tendon Study Group. Clin J Sport Med. 1999;9:129-137.

19. Kaux JF, Forthomme B, Namurois MH, et al. Description of a standardized rehabilitation program based on sub-maximal eccentric following a platelet-rich plasma infiltration for jumper's knee. MLTJ. 2014;4(1):85-89.

20. Visentini PJ, Khan KM, Cook JL, Kiss ZS, Harcourt PR, Wark JD. The VISA score: an index of severity of symptoms in patients with jumper's knee (patellar tendinosis). Victorian Institute of sport Tendon Study Group. J Sci Med Sport. 1998;1:2228.

21. Blazina ME, Kerlan RK, Jobe FW, Carter VS, Carlson GJ. Jumper's Knee. Orthopclin North Am. 1973;4(3):665-678.

22. Filardo G, Kon E, Della Villa S, Vincentelli F, Fornasari PM, Marcacci M. Use of platelet-rich plasma for the treatment of refractory jumper's knee. Int Orthop. 2010;34:909-915.

23. Dragoo JL, Wasterlain AS, Braun HJ, Nead KT. Platelet-rich plasma as a treatment for patellar tendinopathy: a doubleblind, randomized controlled trial. Am J Sports Med. 2014 42(3):610-618.

24. Hoksrud A, Ohberg L, Alfredson H, Bahr R. Ultrasound guided sclerosis of neovessels in painful chronic patellar tendinopathy: a randomized controlled trial. Am J Sports Med. 2006;34: 1738-1746.

25. Housner JA, Jacobson JA, Morag Y, Pujalte GG, Northway RM, Boon TA. Should ultrasound-guided needle fenestration be considered as a treatment option for recalcitrant patellar tendinopathy? A retrospective study of 47 cases. Clin J Sport Med. 2010;20:488-490.

26. Abat F, Diesel WJ, Gelber PE, Polidori F, Monllau JC Sanchez-lbañez JM.Effectiveness of the Intratissue Percutaneous Electrolysis (EPI®) technique and isoinertial eccentric exercise in the treatment of patellar tendinopathy at two years follow-up. MLTJ. 2014;4(2):188-193.

27. Marcheggiani Muccioli GM, Zaffagnini S, Tsapralis K. Open versus arthroscopic surgical treatment of chronic proximal patellar tendinopathy. A systematic review. Knee Surg Sports Traumatol Arthrosc. 2012;21:351-357.

28. Alaseirlis DA, Konstantinidis GA, Malliaropoulos N, Nakou LS, Korompilias A, Maffulli N. Arthroscopic treatment of chronic patellar tendinopathy in high-level athletes. MLTJ. 2013;2(4) 267-272.

29. Creaney L, Hamilton B. Growth factor delivery methods in the management of sports injuries: the state of play. Br J Sports Med. 2008;42(5):314-320.

30. Jo CH, Kim JE, Yoon KS, Shin S. Platelet-rich plasma stimulates cell proliferation and enhances matrix gene expression and synthesis in tenocytes from human rotator cuff tendons with degenerative tears. Am J Sports Med. 2012;40:1035-1045.

31. Gosens T, Oudsten BL, Fievez E, Spijker P, Fievez A. Pain and activity levels before and after platelet-rich plasma injection treatment of patellar tendinopathy: a prospective cohort study and the influence of previous treatments. Int Orthop. 2012;36:1941-1946. 